\title{
Cutting Properties Analysis of Titanium Alloy (Ti-6Al-4V) Based on Cryogenic Cooling
}

\author{
Tang Rui ${ }^{1,2}$, Hou Li ${ }^{*}, 1$, Zhang Qi ${ }^{1,3}$ and Zhou Bo ${ }^{1}$ \\ ${ }^{1}$ School of Manufacturing Science and Engineering, Sichuan University, Chengdu, Sichuan 610065, China \\ ${ }^{2}$ Panxi Technology Innovation Center, Panzhihua University, Panzhihua, Sichuan 617000, China \\ ${ }^{3}$ School of Mechanic Engineering, Panzhihua University, Panzhihua, Sichuan 617000, China
}

\begin{abstract}
This paper studied the cutting properties of Ti-6Al-4V under cryogenic cooling to find a solution for improvement in tool load-carrying, thermal stress, tool life and finish quality. Firstly, this paper established a 3D cutting model of Ti-6Al-4V under cryogenic cooling with liquid nitrogen, and then set the parameters of material, friction model and cutting conditions. Based on this model, the results of chips' shape, cutting forces and temperature distribution were obtained in the simulation with different cooling approaches. The results revealed that although the cryogenic cooling with liquid nitrogen can slightly increase the main cutting force, yet it can efficiently cool the cutting area, and the cooling will contribute to the deformation of chips thereby improving the finish quality.
\end{abstract}

Keywords: Cryogenic, cutting temperature, friction model, Ti-6Al-4V (TC4), tool life.

\section{INTRODUCTION}

Titanium alloy is widely applied in modern advanced equipment manufacturing industry because of its superior comprehensive performance, especially in the aerospace field. But with the features of small thermal conductivity, low modulus of elasticity, strong chemical activity, and easily reacting with surrounding medium under the cutting temperatures, which reduce the cutting performance of titanium alloy greatly, its applications have limitations in many important areas. Now, turning is still the most important method in titanium alloy processing, where many scholars have engaged their researches, and most of the work is focused on the following three aspects: the cutting tool material, cutting parameter optimization and cooling approaches. Researches have shown that the control of cutting temperature is the key to prolong a tool's life, improving its machining efficiency and quality. High pressure and large flow cutting fluid is commonly used in early titanium alloy's cutting to reduce the temperature of cutting area, inhibit the high temperature chemical activity of titanium alloy, and thus prolong tool's life. With the deepening of the research, people found that by reason of titanium alloy's poor thermal conductivity and small cutting contact area, it is easy to bring about stress and thermal concentration. Additionally, since the penetration ability of traditional cutting fluid is poor, so the traditional cooling cannot meet the production requirements with the increase of cutting speed. In recent years, with the introduction of the concept of green manufacturing, many scholars who committed their research to strong air cooling cutting, low temperature freezing cutting, liquid nitrogen (LN2) cooling cutting, MQL (Minimal Quantity Lubrication)and other new

*Address correspondence to this author at the School of Manufacturing Science and Engineering, Sichuan University, Chengdu, Sichuan 610065, China; Tel: +86-13880471291; E-mail: Houli4@163.com types of cooling methods, have achieved positive results. The LN2 cooling with its good cooling and lubricating effect and environmental-friendly character has attracted attention from many researchers.

Research on LN2 cooling cutting TC4 (Ti-6A1-4V) has started earlier. Hong $[1,2]$ and other scholars studied the effects of different cooling locations in the cutting process of titanium alloy with LN2. They found that the main cutting edge with a local concentration of liquid nitrogen cooling can give a better result of temperature control. However, due to changes in the hardness and strength of the material under the strong cooling conditions, the cutting force is slightly increased compared to dry cutting. Pradeep kumar used a special tool with liquid nitrogen nozzle for TC4 cutting experiment, and the results showed that compared with water base cutting fluid, LN2 cooling can reduce the temperature by $61-66 \%$, whereas lower cutting force by $35-42 \%$ [3]. Domestic scholars Zhao Wei and Meng Chun also researched on the cutting properties of titanium alloy under liquid nitrogen cooling condition $[4,5]$. The feasibility of finite element method (FEM) in cutting simulation has been proved by many scholars, but the simulation of TC4 cutting under LN2 cooling conditions has been rarely studied due to its complex relationship of thermodynamics and heat transfer boundary definition. Hong, $\mathrm{Li}$ Wei and other scholars established robust two-dimensional finite element model of orthogonal cutting of titanium alloy in their respective research, but there are limitations. The finite element model of Hong et al. has a perfect heat transfer boundary setting and experimental basis, but since it does not contain material constitutive model, the cutting force and other data is measured directly by the experiment. On the other hand, the finite element model of Li Wei et al has no experimental data to prove its correctness, besides the two-dimensional finite element model of orthogonal cutting simulation has some limitations. 
This paper puts forward a three-dimensional finite element model considering the heat transfer characteristics of cutting, frictional properties of LN2 cooling conditions. Based on this model, we compared the influence of cutting force and tool temperature with that of different cooling parts of the cutting area (cooling rake face and flank face respectively). The establishment of this model can provide a prediction reference for the cutting forces, temperature and frictional properties of TC4 cutting under LN2 cooling, and foundation for further simulation research, such as temperature, surface residual stress and tool wear.

\section{THE ESTABLISHMENT OF THE TITANIUM ALLOY CUTTING MODEL WITH LN2 COOLING}

A correct finite element model depends on a proper meshing size, accurate material constitutive equation, and a good definition of thermal boundary conditions, see ref $[1,4$, 6]. This paper established a three-dimensional finite element model with Deform-3D.

\subsection{D Model and Mesh}

3D model of the tool and the workpiece we used are shown in Fig. (1), INSERTS CNMA432, WC and uncoated. The MCLNR tool holder is used to create a geometrical configuration of the inclination angles of the major cutting edge and the minor cutting edge $\mathrm{i}=\mathrm{i}^{\prime}=-5^{\circ}$, insert nose angle $\delta=80^{\circ}$, and side angle of the major cutting edge $\theta=95^{\circ}$, which is widely used in the United States Air titanium machining. Based on literature [7], tool mesh number is 155023 , rigid, tetrahedron, minimum grid side length is 0.01 $\mathrm{mm}$. Workpiece material is considered as plastic perfect, with the grids for 136,630 , and the minimum mesh side length is $0.03 \mathrm{~mm}$.

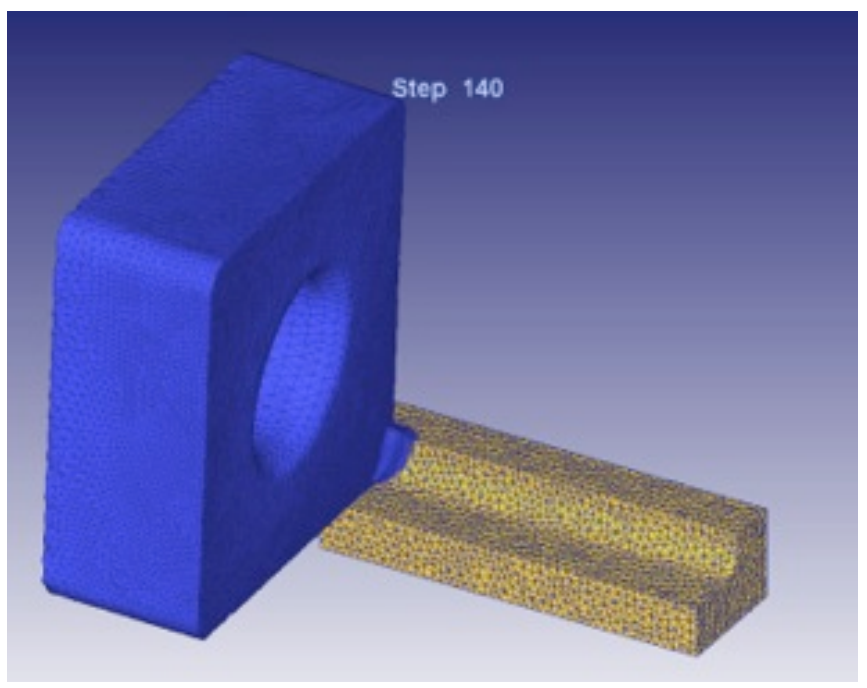

Fig. (1). 3D model of the blade and the workpiece and meshing.

\subsection{Material Parameters and Constitutive Model}

When cutting titanium alloy, the temperature is relatively low and material deformation rate is high in the first strain zone. In the second strain zone, on the other hand, although the material strain rate decreased, yet the tool-chip contact usually generated high temperatures and concentrated stress which results in a big gradient of temperature and stress in the deformation zone. Therefore, the material constitutive model should reflect strain, strain rate, the influence of temperature on the material yield stress and flow stress. Researchers have shown that Johnson - Cook constitutive equation can reach a good agreement with the experiment when simulate the cutting process of TC4, which is shown in equation (1):

$\sigma=\left(C_{1}+C_{2} \varepsilon^{n}\right) \cdot\left[1+C_{3} \ln \left(\frac{\dot{\varepsilon}}{\dot{\varepsilon}_{0}}\right)\right]\left[1-\left(\frac{T-293}{1625}\right)^{m}\right]$

The parameters are set according to the literature [7], that is the yield strength of the $C_{1}=850 \mathrm{MP}$, stress constant $C_{2}$ $=350 \mathrm{MP}$, material hardening exponent $\mathrm{n}=0.16$, strain rate hardening coefficient $C_{3}=0.025$, thermal softening coefficient $\mathrm{m}=0.5$. Where: $\sigma$ is the material flow stress, $\varepsilon$ is the strain, $\dot{\varepsilon}$ is strain rate, $\dot{\varepsilon_{0}}$ is reference strain rate, and set its value to 1 . In this study, the thermal conductivity and heat capacity of Ti-6A1-4V changed greatly with temperature; the calculation relationship curve is shown in Fig. $(\mathbf{2 a}, \mathbf{b})$.

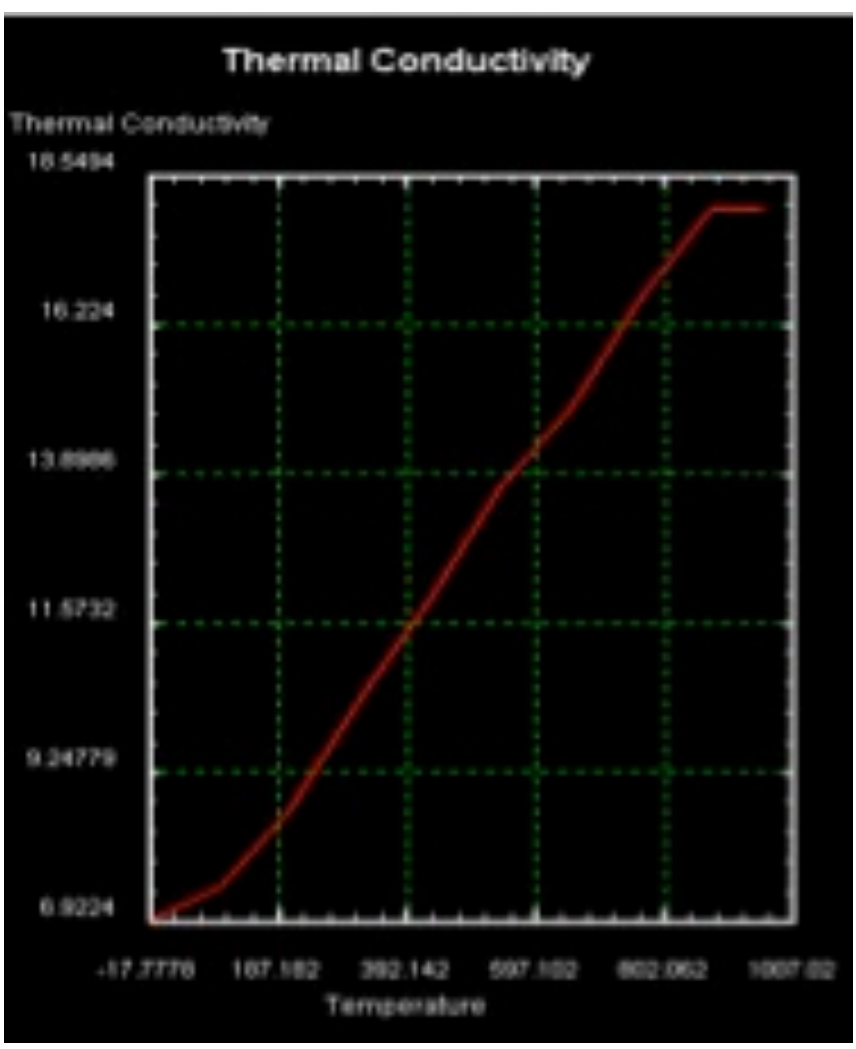

Fig. (2a). Curve of thermal conductivity change.

\subsection{D Heat Transfer Equation and Thermal Boundary Definition}

When machining TC4, chips continuously separated from the blank body, which the Deform-3D achieves the chip separation through re-meshing process. The heat conduction in the cutting progress is governed by a $3 \mathrm{D}$ heat transfer equation, expressed as follows:

$\left(\frac{\partial}{\partial \mathrm{x}}\left(\mathrm{k}_{\mathrm{x}} \frac{\partial \mathrm{T}}{\partial \mathrm{x}}\right)+\frac{\partial}{\partial \mathrm{y}}\left(\mathrm{k}_{\mathrm{y}} \frac{\partial \mathrm{T}}{\partial \mathrm{y}}\right)+\frac{\partial}{\partial \mathrm{z}}\left(\mathrm{k}_{\mathrm{z}} \frac{\partial \mathrm{T}}{\partial \mathrm{z}}\right)\right)+\dot{\mathrm{q}}=\rho c \frac{\partial \mathrm{T}}{\partial \mathrm{t}}$ 
where $\mathrm{T}$ is the object's temperature, $\dot{\mathrm{q}}$ represents strength of the heat source produced by a volume unit inside the object within a time unit, $\rho$ is material density, $\mathrm{c}$ is specific heat, $\mathrm{k}_{\mathrm{x}}, \mathrm{k}_{\mathrm{y}}, \mathrm{k}_{\mathrm{z}}$ are the thermal conductivity in the three axial directions respectively.

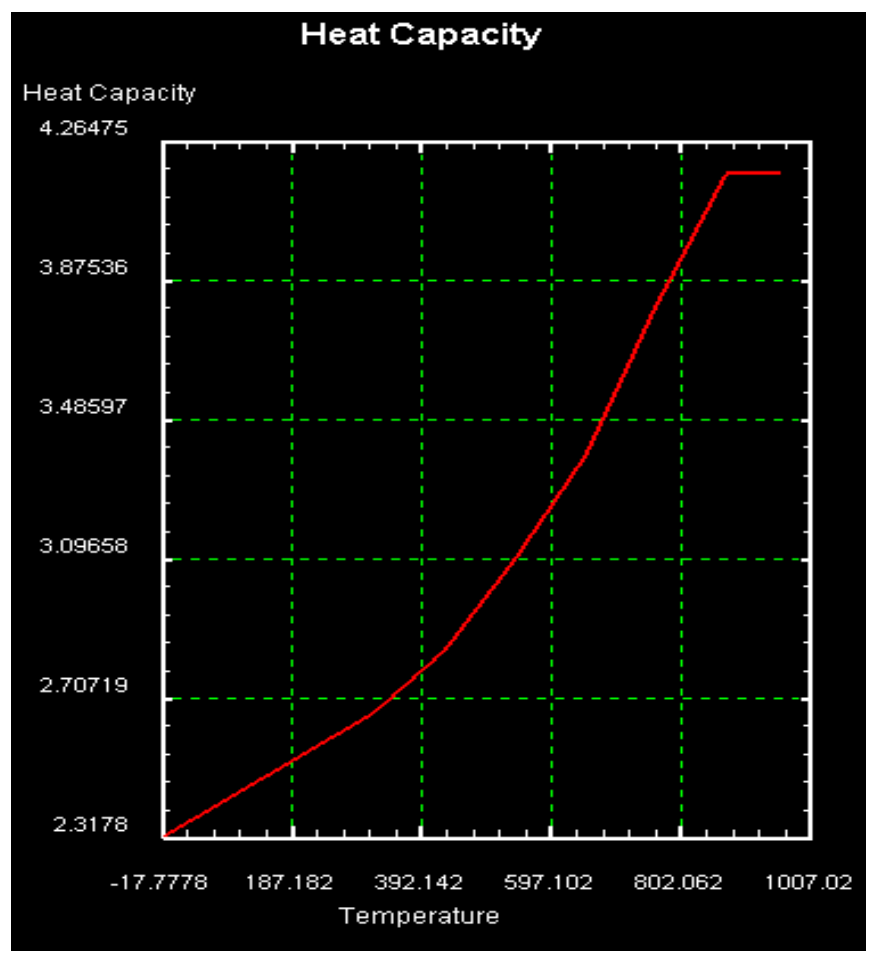

Fig. (2b). Curve of heat capacity change.

Thermal boundary definition is of vital importance in this study. On the basis of previous studies, this paper gives the following definition: boundary ABCDEFGHIJKLA, as shown in Fig. (3) can be divided into four different thermodynamic boundaries: $T_{r}$ (constant temperature boundary), $T_{a}$ (air convection boundary), $T_{n}$ (liquid nitrogen cooling boundary), and $T_{i}$ (adiabatic boundary).

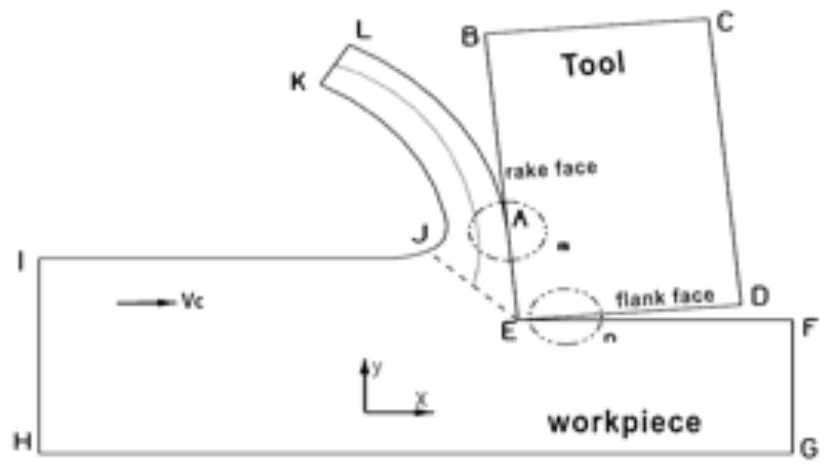

Fig. (3). The model of thermodynamic boundary.

Dry cutting, the boundary conditions are defined as follows:

$$
\left\{\begin{array}{r}
\text { GH、HI } \in T_{r} \\
\text { KL、BC、CD、FG } \in T_{i} \\
\text { IJ、JK、LA AB、ED、EF } \in T_{a}
\end{array}\right.
$$

For the rake surface with LN2 cooling cutting, the boundary conditions are defined as follows: $\left\{\begin{array}{r}\text { GH、HI } \in T_{r} \\ \text { LA } 、 \mathbf{A B} \in T_{n} \\ \text { IJ、JK、 DE、EF } \in T_{\boldsymbol{a}} \\ \text { KL、BC、 CD、FG } \in \boldsymbol{T}_{\boldsymbol{i}}\end{array}\right.$

For the flank surface with LN2 cooling cutting, the boundary conditions are defined as follows:

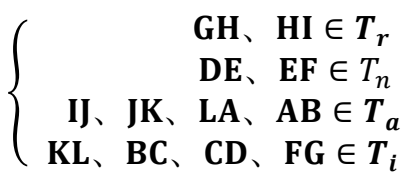

Since this study takes the tool as a rigid body, so we need to just consider the heat transfer parameters of the tool material in contact with the chip as well as its own thermal conductivity and so on.

\subsection{The Friction Model of the Tool Chip Contact}

The frictional condition in the cutting area within tool surface contact with the chip and work piece is very complex, because of the stress and temperature concentration. At present, the majority of scholars have usually divided it into slide area and bonded area, using the Zorve [8] friction model for numerical calculation, specifically as follows:

$\tau_{\text {fr }}=\left\{\begin{array}{cc}\delta \sigma_{n}, \tau_{\mathrm{fr}}<\bar{\tau}_{\max } & \text { (slide area) } \\ \bar{\tau}_{\max }, \tau_{\mathrm{fr}} \geq \bar{\tau}_{\max } & \text { (bonded area) }\end{array}\right.$

where: $\tau_{f r}$ is the friction stress of the contact area, $\sigma_{n}$ is the normal stress, $\bar{\tau}_{\max }$ is the workpiece material critical shear yield strength, and $\delta$ is the coefficient of friction stress. According to the literature [6], take $\delta=0.82$. The contact between chip and tool rake face largely affects the tool temperature, and heat transfer coefficient between the two directly determines the tool temperature calculation, thereby affecting the tool's life and cutting forces. In the finite element simulation, some scholars put forward that in order to reach thermal equilibrium quickly, it needs to change the heat transfer coefficient between the tool and the chip [7-10]. According to literature [9], it is in good agreement with the experimental results when the heat transfer coefficient is set to $2000 \mathrm{KW} /\left(\mathrm{m}^{2} \cdot \mathrm{K}\right)$.

\section{SIMULATION RESULTS AND DISCUSSION}

\subsection{Differences in Serrated Chip}

On the basis of 3D cutting model proposed in this paper, the cutting parameters are: cutting speed $\mathrm{V}_{\mathcal{C}}=90 \mathrm{~m} / \mathrm{min}$, feed rate $\mathrm{f}=0.254 \mathrm{~mm} / \mathrm{rev}$, and back engagement $\mathrm{a}_{\mathrm{p}}=0.254 \mathrm{~mm}$. Simulation of serrated chip, as shown in Fig. (4), is caused when the entire first deformation zone slips and where thermoplastic instability occurs to form a zigzag structure, which is common in the cutting of difficult-to-machine materials such as titanium alloy. Simulation of dry cutting serrated chip is shown in Fig. (4a), whereas liquid nitrogen cooled rake and flank face are respectively shown in Fig. $(\mathbf{4 b}, \mathbf{c})$. It can be seen that under the effect of liquid nitrogen cooling, the serrated structure of chip becomes more intensive than by dry cutting. 


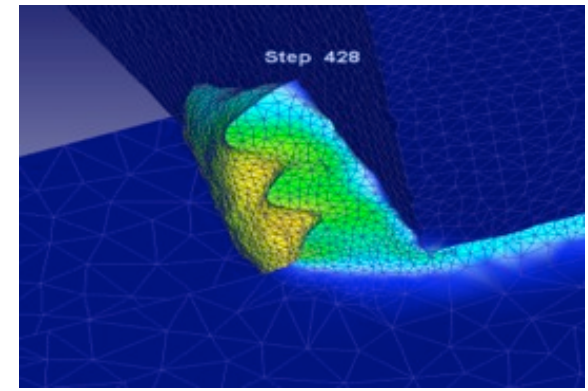

Fig. (4a). Dry cutting jagged structure.

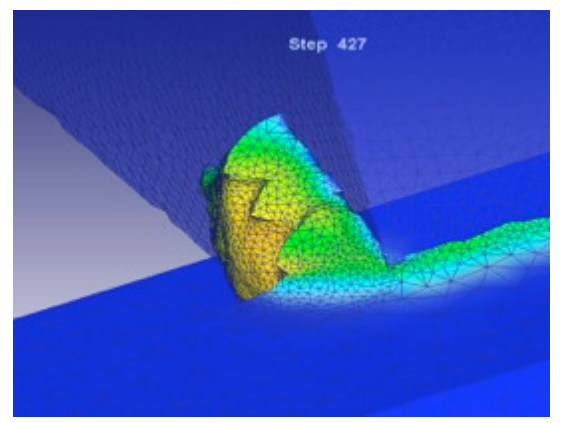

Fig. (4b). Flank face cooling jagged structure.

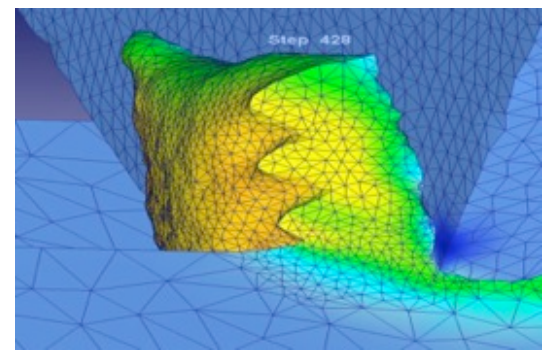

Fig. (4c). Rake face cooling jagged structure.

\subsection{Analysis of the Cutting Force}

Even if the cutting force reaches steady state, there is still a certain degree of instability, as shown in Fig. (5). It can be seen from the graph that the LN2 cooling has no obvious influence on the cutting force, and the main cutting force has a tendency to rise due to the change in the strength of the titanium alloy affected by cooling.

In order to compare, we concluded the average main cutting force under different cooling ways after reaching steady state: Dry Cutting $\mathrm{F}=808.4 \mathrm{~N}$, rake liquid nitrogen cooling $\mathrm{F}=891 \mathrm{~N}$, flank liquid nitrogen cooling $\mathrm{F}=916 \mathrm{~N}$, and these tendencies coincide with Hong's experimental results [2].

\subsection{Analysis of the Tool Temperature}

The temperature distributions in the simulation step 350, respectively for different cooling modes of the tool, are shown in Fig. (6). As we can see from the graph, the difference between the maximum temperatures with different cooling methods is small; respectively $\mathrm{T}_{\text {dry }}=510^{\circ} \mathrm{C}$ $\mathrm{T}_{\text {flank }}=478^{\circ} \mathrm{C}, \mathrm{T}_{\text {rake }}=470^{\circ} \mathrm{C}$, which is within the range of $7 \%$ compared with each other. The reason is that in a small tool-chip contact area, for the high pressure and high contact stresses, the sprayed liquid nitrogen cannot penetrate the area to achieve effective cooling.

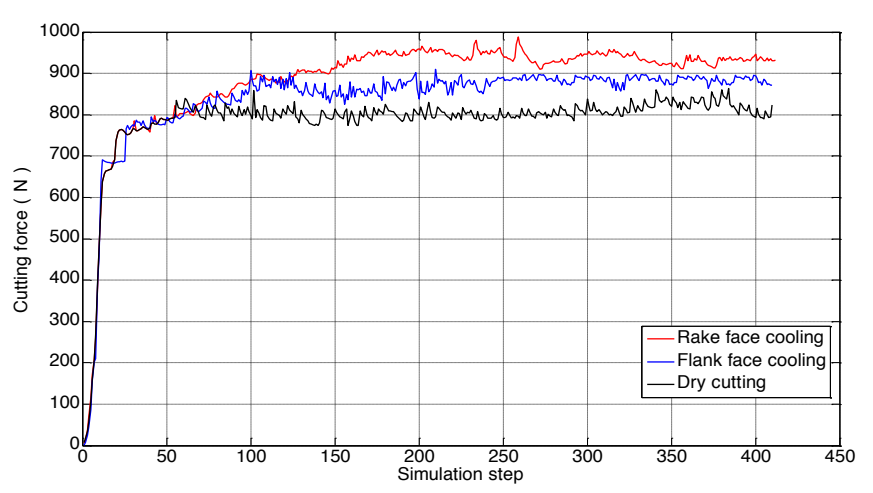

Fig. (5). Steady cutting force.

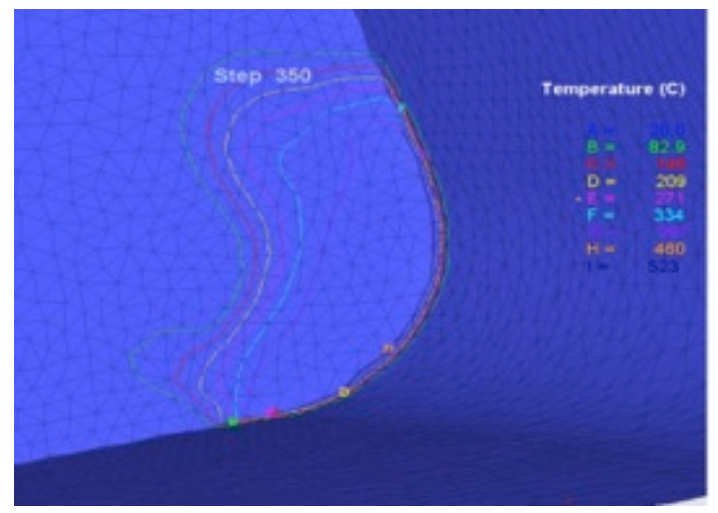

Fig. (6a). The temperature distribution of the dry cutting tool.

Fig. (6d) is the tool average temperature bar chart created under different cooling methods. It can be seen that the liquid nitrogen cooling compared to dry cutting can greatly reduce the average temperature of the cutting tool. The rake cooling can reduce the average temperature of the cutting tool by $47 \%$, whereas the flank cooling, by $39 \%$. This data is perfectly coincide with Hong`s [1] experimental results.

\section{CONCLUSION}

From the analysis of the simulation results, we can get the following conclusions:

The three-dimensional cutting model established in this paper is feasible in the treatment of thermal boundary and selection of parameters. The simulation results and experiment are consistent, laying the foundation for further three-dimensional cutting simulation.

Under the effect of liquid nitrogen cooling, serrated chips produced by cutting TC4 compared to dry cutting are more intensive, which is conducive to the chip curl and chip breaking, reducing friction between the chip and the rake face, helping improve the surface quality.

The cutting force increases slightly when the titanium alloy is cooled by outside spraying of liquid nitrogen. Since the cooling leads to changes in material intensity, its main cutting force increases slightly compared to dry cutting, where rake face cooling rises by $10.2 \%$, flank face cooling rises by $13.3 \%$.

Although the liquid nitrogen cooling can effectively reduce the average temperature of the tool, and the cooling effect on rake face is better than flank face, yet the maximum temperature of the surface is less affected. 


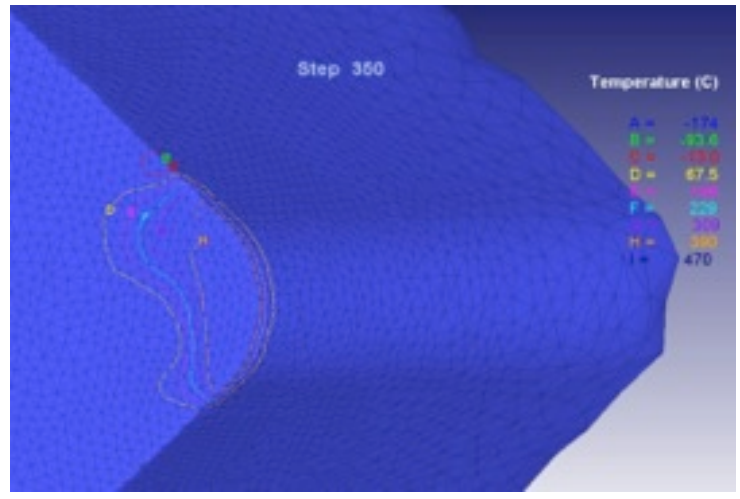

Fig. (6b). The temperature distribution of rake face liquid nitrogen cooling.

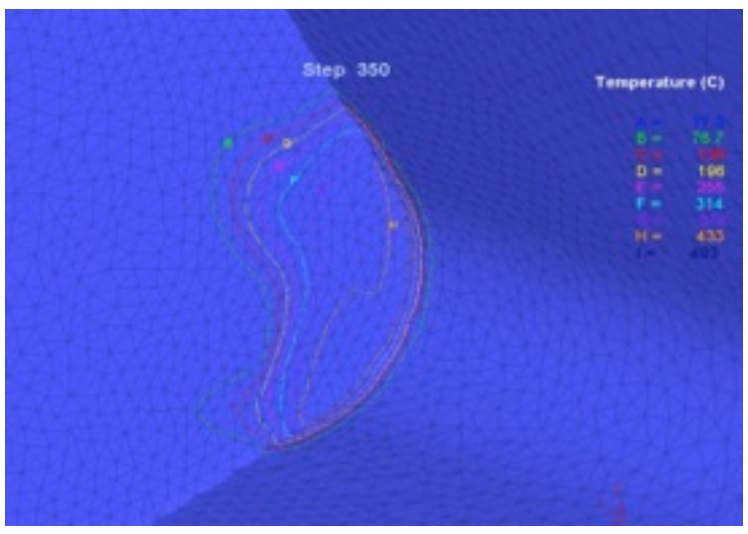

Fig. (6c). The temperature distribution of flank face liquid nitrogen cooling.

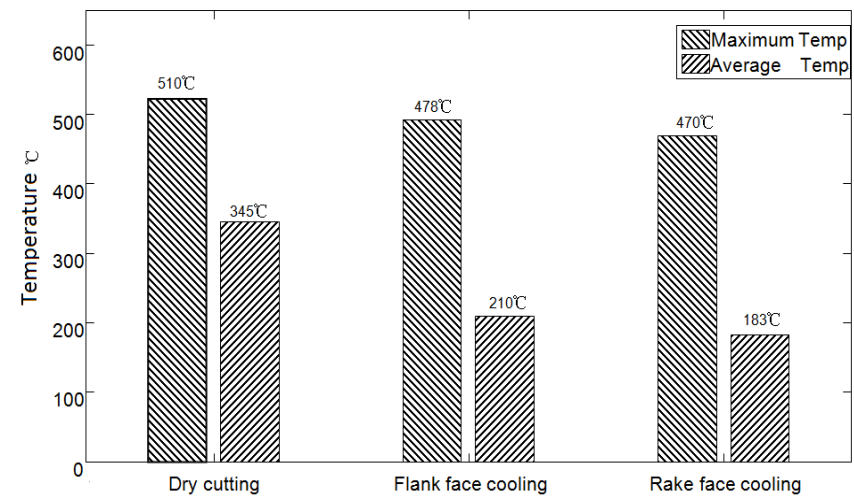

Fig. (6d). The highest temperature and the average temperature of cutting zone.

\section{CONFLICT OF INTEREST}

The authors confirm that this article content has no conflict of interest.

\section{ACKNOWLEDGEMENTS}

This work was supported by the key laboratory of vanadium and titanium resources comprehensive utilization of Sichuan Province (No. 2013ZD-04), Panzhihua city (No. 2013TX -8), and Panzhihua University (No. 2014YB017).

\section{REFERENCES}

[1] SY Hong, Y Ding, Cooling approaches and cutting temperatures in cryogenic machining of Ti-6Al-4V. Inter $\mathrm{J}$ Machine Tools \& Manufacture 2001; 41(10): 417-1437.

[2] SY Hong, Y Ding, and W Jeong. Friction and cutting forces in cryogenic machining of Ti-6Al-4V. Inter $\mathrm{J}$ Machine Tools \& Manufacture 2001: 41(15): 2271-85.

[3] M Dhananchezian, MP Kumar. Cryogenic turning of the Ti-6Al-4V alloy with modified cutting tool inserts. Cryogenics 2011; 51(1): 34-40.

[4] W Zhao, N He, L Li.Finite element analysis of orthogonal cutting of Ti6Al4V alloy in enhanced cooling condition. J South China University of Technology 2006; 34(7): 40-4.

[5] W Zhao, N He, L Li, Z Man. Experimental study on high speed milling of Ti-6Al-4V alloy with nitrogen-oil-mist. Journal of Nanjing University of Aeronautics and Astronautics, 2006; 38(5): 634-8.

[6] A Attanasio, E Ceretti, A Fiorentino, C Cappellini, C Giardini, Investigation and FEM-based simulation of tool wear in turning operations with uncoated carbide tools. Wear 2010; 269: 335-44.

[7] Y Chen, S Yang, Y Fu, J Xu, H Su. FEM estimation of tool wear in high speed cutting of Ti6Al4V alloy. Acta Aeronautica Et Astronautica Sinica 2013; 34(9): 2230-40.

[8] N N Zorev. Inter-relationship between shear processes occurring along tool face and shear plane in metal cutting. In: International Research in Production Engineering ASME New York 1963; 42-49.

[9] L Filice, M Umbrello, F Micari. FE analyses of tool wear in orthogonal cutting. In: Proceedings of the $2^{\text {nd }}$ International Conference on Tribology in Manufacture Process 2004; 187-194.

[10] B Shu, N He, and K Wu. Study on the milling of Ti alloy in nitrogen gas medium. Aviation Precision Manufacturing Technology. 2002; 38(6): 16-19.

(C) Rui et al.; Licensee Bentham Open.

This is an open access article licensed under the terms of the Creative Commons Attribution Non-Commercial License (http:/creativecommons.org/licenses/ by-nc/4.0/) which permits unrestricted, non-commercial use, distribution and reproduction in any medium, provided the work is properly cited. 